\title{
The first spectroscopic detection of rapid multi-mode pulsations in Przybylski's star
}

\author{
D.E. Mkrtichian ${ }^{1,2}$ and A.P. Hatzes ${ }^{3}$ \\ ${ }^{1}$ Astrophysical Research Center for the Structure and Evolution of the Cosmos, Sejong \\ University, Seoul, 143-747, (South) Korea \\ ${ }^{2}$ Astronomical Observatory, Odessa National University, Shevchenko Park, Odessa, 65014, \\ Ukraine \\ email: david@arcsec.sejong.ac.kr \\ ${ }^{3}$ Thüringer Landessternwarte, Tautenburg, Sternwarte 5, D-07778, Germany \\ email: artie@tls-tautenburg.de
}

\begin{abstract}
Based on a preliminary analysis of 3 nights of high-dispersion spectral observations of HD 101065 (Przybylski's star) obtained on the ESO 3.6-m telescope + HARPS we find rapid multiperiodic radial velocity oscillations of at least three pulsation modes. The detailed analysis of the frequency space and probable splitting based on 4 nights of data will be given later.
\end{abstract}

Keywords. Stars: oscillations, stars: chemically peculiar, stars: individual: (HD 101065)

\section{Introduction}

The peculiar nature of HD 101065 (Przybylski's star) was discovered by Przybylski (1961) and was found to be the most chemically peculiar star in the sky. The star shows strong (up to +4 dex) anomalies of the lanthanides (Cowley et al. 2000), technecium, and promethium (Cowley 2003, Cowley et al. 2004). The recent abundance analyses of Przybylski's star (Gopka et al. 2005, Bidelman 2004) possibly show the presence of spectral lines from other radioactive actinides.

Kurtz (1980) discovered short-period pulsations in Przybylski's star establishing it as a rapidly oscillating Ap (roAp) star. This star oscillates photometrically with 3 frequencies centered on the principal mode of 12.1 minutes (Martinez \& Kurtz 1990). The authors tentatively suggested a spacing of $57.8 \mu \mathrm{Hz}$. We present the first spectroscopic detections of rapid pulsations in Przybylski's star.

\section{Observations and reduction}

Spectral observations of HD 101065 were made on 4 consecutive nights (3-6 March 2004) using the HARPS echelle-spectrograph of the 3.6-m telescope of ESO. The wavelength coverage was $\lambda \lambda 3800-6900$ at a resolving power $R=100000$. Exposure times were typically 70-80 s. Precise stellar radial velocities measurements were made using an iodine cell placed just before the entrance fiber of HARPS. As of this writing we were still in the process of reducing the data. Thus, only 3 nights of radial velocity (RV) measurements were analyzed.

To achieve the maximum RV measurement precision we used the full spectral region covered by iodine reference absorption lines. This produces an "integrated" RV value for the spectral region covered by iodine absorption lines (approximately $\lambda \lambda 5100-5900)$. To determine the relative RV for HD 101065 each spectral order was divided into 20 sections so that we could model any possible spatial and temporal variations of the instrumental 
Table 1. The oscillation spectrum of HD 101065

\begin{tabular}{lll}
\hline Mode & $\begin{array}{l}\text { Frequency } \\
(\mathrm{mHz})\end{array}$ & $\begin{array}{l}\text { Mean semi-amplitude } \\
\left(\mathrm{m} \mathrm{s}^{-1}\right)\end{array}$ \\
\hline$\nu_{1}$ & 1.373 & $221($ var $)$ \\
$\nu_{2}$ & 1.324 & $16($ var $)$ \\
$\nu_{3}$ & 1.422 & 8 (var $)$ \\
\hline
\end{tabular}
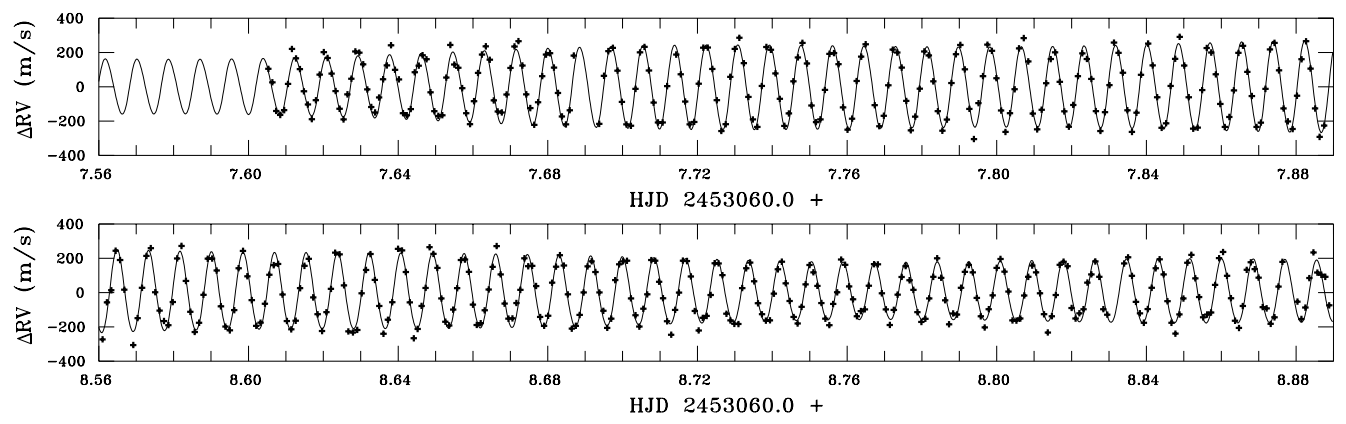

Figure 1. The pulsational RV variation of HD 101065 measured over the whole wavelength range $\lambda \lambda 5100-5900$ or 3 Mar 2004 (top) and 4 Mar 2004 (bottom)

profile using the procedure of Valenti at al. (1995). A mean weighted average over the sections was used for final RV measurements. Figure 1 shows the RV curves of first two nights (3, 4 March 2004). The solid line is the multifrequency fit.

\section{Results}

A preliminary time series analysis was performed on the RV measurements which span the first three nights. We find a multiperiodic RV variations related to the three principal modes. We see amplitude modulation of the individual modes over the interval of the observations. The frequencies and mean amplitudes of individual modes are given in the Table 1 . The accurate frequency values and the rotation period should be revealed in analysis of complete 4-day interval and is in progress.

\section{Acknowledgements}

Work by DM is a part of research activity of the Astrophysical Research Center for the Structure and Evolution of the Cosmos (ARCSEC) that is supported by Korea Science and Engineering Foundation (KOSEF) through the Science Research Center (SRC) program. APH acknowledges the support of grant 50OW0204 from the Deutsches Zentrum für Luft- und Raumfahrt e.V. (DLR).

\section{References}

Bidelman, W.P 2004, private communication

Cowley, C. 2003, http://astro.1sa.umich.edu/users/cowley/

Cowley, C., Ryabchikova, T., Kupka, F., Bord, D.J., Mathys, G., \& Bidelman, W.P. 2000, MNRAS 317, 299

Cowley, C.R., Bidelman, W.P., Hurbig, S., Matis, G., \& Bord, D.J. 2004, A\&A 419, 1087

Gopka V.F., Yushchenko A.V., Shavrina A.V., Mkrtichian D.E., Hatzes A.P., Andrievky S.M., Cherysheva L.V. 2004, These proceedings, FP18

Kurtz, D.W. 1980, MNRAS, 190, 103 
Martinez P. \& Kurtz D.W. 1990, MNRAS 242, 636

Przybylski, A. 1961, Nature 189, 4766, 739

Valenti, J. A., Butler, R. P., \& Marcy, G. W. 1995, PASP 107, 966 